Archives de sciences sociales des religions

$168 \mid 2014$

Bulletin Bibliographique

\title{
Nicolas Balzamo, Les deux cathédrales. Mythe et histoire à Chartres $\left(\mathrm{XI}^{\mathrm{e}}-\mathrm{XX}^{\mathrm{e}} \mathrm{s}\right.$.)
}

Paris, Les Belles Lettres, coll. « Vérité des mythes », 2012, 384 p.

\section{Alain Rauwel}

\section{CpenEdition}

\section{Journals}

Édition électronique

URL : http://journals.openedition.org/assr/25861

DOI : 10.4000/assr.25861

ISSN : 1777-5825

Éditeur

Éditions de l'EHESS

Édition imprimée

Date de publication : 31 décembre 2014

Pagination : 117

ISBN : 978-2-7132-2467-6

ISSN : 0335-5985

Référence électronique

Alain Rauwel, « Nicolas Balzamo, Les deux cathédrales. Mythe et histoire à Chartres ( $\mathrm{xl}^{\mathrm{e}-\mathrm{xx}} \mathrm{e}^{\mathrm{e}} \mathrm{s}$.) »,

Archives de sciences sociales des religions [En ligne], 168| 2014, mis en ligne le 31 mars 2015, consulté le 22 septembre 2020. URL : http://journals.openedition.org/assr/25861 ; DOI : https://doi.org/ 10.4000 /assr. 25861

Ce document a été généré automatiquement le 22 septembre 2020

(c) Archives de sciences sociales des religions 


\section{Nicolas Balzamo, Les deux cathédrales. Mythe et histoire à Chartres $\left(\mathrm{XI}^{\mathrm{e}}-\mathrm{XX}^{\mathrm{e}} \mathrm{s}\right.$.)}

Paris, Les Belles Lettres, coll. «Vérité des mythes », 2012, 384 p.

\section{Alain Rauwel}

\section{RÉFÉRENCE}

Nicolas Balzamo, Les deux cathédrales. Mythe et histoire à Chartres ( $\mathrm{XI}^{\mathrm{e}}-\mathrm{XX}^{\mathrm{e}} \mathrm{s}$.), Paris, Les Belles Lettres, coll. « Vérité des mythes », 2012, 384 p. 
1 Le terrain immense et fascinant des origines imaginaires a été exploré en tous sens ces derniers temps, dans le sillage de deux grands livres: l'essai d'A. Remensnyder sur les récits de fondations monastiques et celui de R. Bizzocchi sur les "généalogies fabuleuses ». Dans ce qui est désormais une tradition prend place N.Balzamo, avec pour objet un morceau de choix : le mythe de la Virgo paritura, cette connaissance mystérieuse qu'auraient eue les druides du pays des Carnutes de l'Incarnation à venir et qui ferait de Notre-Dame de Chartres le plus ancien sanctuaire chrétien qui soit. Comme le dit très justement l'auteur, il y a là un monument narratif et textuel aussi impressionnant que la célébrissime

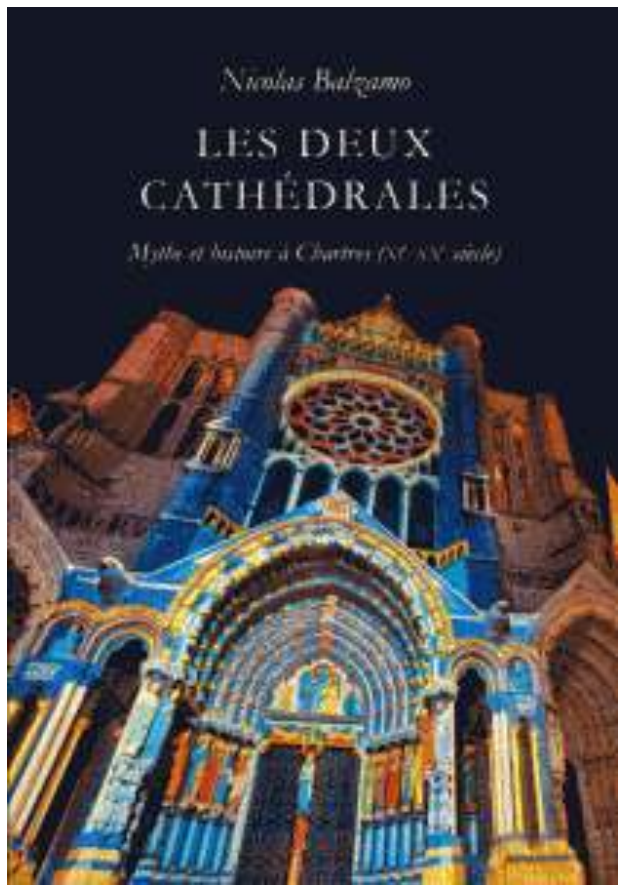
cathédrale de pierre qui attire à Chartres

d'innombrables visiteurs. Rien de plus judicieux, par conséquent, que d'en entreprendre l'archéologie. On peut en effet espérer, en rassemblant soigneusement toutes les pièces du dossier, écrire rien moins que «la vie et la mort d'une vérité ». A. Sanfaçon avait déjà, en plusieurs riches études, apporté de précieux éléments en ce sens, que N. Balzamo écarte sans doute un peu vite pour avancer son propre système mais c'est de bonne guerre!

2 Pour que le mythe " prenne ", il faut un faisceau d'éléments, fort bien présentés ici. Le substrat matériel est indispensable ; il prend à chartres la double forme d'une relique et d'une image. La relique est celle de la «sainte chemise ", qui aurait été donnée à la cathédrale par Charles le Chauve et qui a joué un grand rôle dans la réputation chartraine. L'image est celle de Notre-Dame Sous-Terre, inséparable d'un environnement d'autant plus séduisant qu'il était chtonien, avec ses circulations spécifiques et ses lieux énigmatiques, comme le puits des Saints-Forts. À ces objets cultuels se surimpose un discours, inscrit non dans l'espace urbain, ni même dans l'espace diocésain, mais bien dans ce cadre décisif, surtout en France capétienne, qu'était la province ecclésiastique. C'est à partir de l'invention des reliques des saints Savinien et Potentien à Sens que se développe tout un récit des origines apostoliques, extrêmement banal en soi, mais porté au rouge par l'introduction de la Vierge ellemême, vénérée et reconnue comme souveraine à Chartres. Et le mythe de s'enrichir d'ambassades à Nazareth, de lettres de la Madone et autres singularités, non dénuées d'impact sur les pouvoirs urbains et les revendications attachées à leur exercice. N. Balzamo suit l'étonnant gonflement de cet objet narratif initialement très ténu avec rigueur et limpidité. Dans la mesure cependant où il connaît parfaitement, comme le montre sa bibliographie, l'ensemble des mythes jumeaux ou cousins de celui de Chartres (celui des saints de Provence par exemple), on eût aimé qu'il développât la dimension comparative de son analyse. Nul doute que quelques excursions au-delà des 
horizons un peu plats de la Beauce eussent introduit dans l'ouvrage une heureuse variété.

3 Reste que la grande richesse des Deux cathédrales est l'amplitude de leur spectre chronologique. En véritable historien, l'auteur se fait tour à tour médiéviste et moderniste. L'Ancien Régime, précisément, est le temps des premières remises en cause. L'érudition moderne achoppe sur les fantaisies chartraines. Il est particulièrement remarquable que la première critique savante du mythe émane d'un chanoine de Chartres, J.-B. Souchet (1654), et il est plus remarquable encore que le même, parallèlement à son travail sur les textes, soit le responsable d'un remodelage complet des lieux, la chapelle souterraine prenant un tout autre visage au mitan du $\mathrm{XVII}^{\mathrm{e}}$ siècle. Travail de cabinet, aménagements liturgiques et implications pastorales vont du même train, comme on le note aussi au Siècle des Lumières lorsque Chartres, tardivement, réforme son bréviaire et en déniche vieux saints et vieilles histoires.

Comme le souligne l'auteur, on pouvait s'attendre à ce que l'histoire s'arrêtât là. Mais on se heurte, à Chartres comme ailleurs, au fascinant problème des revivals du XIX siècle. Ce que ni évêque ni chanoines n'eussent osé chuchoter en 1760 , le prélat chartrain le clame en 1860 : «c'est un fait appuyé sur une tradition constante que les druides honoraient à Chartres la Vierge »! Et malheur aux historiens, en ce contexte : on connaît les fulminations de l'archevêque de Sens contre l'abbé Duchesne, coupable entre autres d'avoir renvoyé sèchement les prétentions du clergé chartrain à leur néant scientifique. Le lecteur sensible à ces aspects trouvera certainement que Balzamo va trop vite, beaucoup trop vite, quand il traite des périodes postrévolutionnaires. Ce ne sont pas des épiphénomènes tardifs et condamnés par avance qu'il a à évoquer, mais, en profondeur, des conceptions philosophiques, anthropologiques et politiques qui rejoignent les débats les plus vifs de l'Europe entre romantisme et scientisme. Des chapitres bien plus amples eussent été nécessaires, d'une part pour mettre en valeur l'impact de 1870 (Mgr Pie parle du «sanctuaire de Marie le plus national» en 1873, donnant au culte de la Vierge la même dimension réparatrice qu'au Sacré-Cœur qui triomphe alors à Paray ou à Montmartre et faisant de Chartres un équivalent de ce que sera Le Puy en 1942), d'autre part pour développer la dimension littéraire du mythe au temps du " catholicisme des écrivains ». Trois lignes sont consacrées à Huysmans là où il faudrait plusieurs pages, tant il est vrai qu'à la toute fin du cycle analysé par l'auteur les deux cathédrales deviennent trois : l'église de pierre, le complexe hagiographicoliturgique et la cathédrale romanesque. Les historiens de la littérature et des arts ont bien montré la place capitale prise par l'objet-cathédrale dans l'imaginaire de la génération de 1880, il n'eût pas été difficile de faire fond sur ces travaux dans le cas particulier de la Virgo paritura. Et quelle déception, encore, de voir le virage ésotérisant du légendaire marial réduit à une petite note de fin! L'infrahistoire, certes négligeable du strict point de vue de l'apport objectif, est une extraordinaire fenêtre ouverte sur les conceptions et les aspirations de milieux plus larges qu'on ne le croit souvent, et appelle comme telle tout l'intérêt des chercheurs. Markale doit être examiné comme l'ont été Duparc, Roulliard ou Savard.

Les regrets que l'on peut éprouver par endroits ne mettent que mieux en lumière la qualité et même l'exemplarité de l'enquête menée par Nicolas Balzamo. Ambitieuse, solide, ouverte sur un bel essai final d'« interprétation générale », elle sera désormais indispensable à tous ceux qui cherchent à comprendre comment, à l'intérieur de la «métamythologie » chrétienne, de grands récits localisés émergent, s'affirment... et 
disparaissent sur la très longue durée de l'histoire des faits de croyance que l'on pourra continuer, si l'on veut, à qualifier de « long Moyen Âge ». 